En aquel entonces la cocina ecuatoriana tradicional cumplía un rol social; es decir, permitía la participación no solamente de miembros de la familia, sino también de amigos y de otro tipo de invitados. La cocina actual, sin embargo, ya no posee las mismas condiciones de aplicación que la cocina tradicional, ni respecto al proceso de preparación de alimentos ni respecto al tiempo disponible para compartir en torno a la mesa con la misma cercanía y distención.

Ahora bien, dadas estas variaciones en las condiciones actuales de vida, se torna indispensable destacar la necesidad de un conocimiento a fondo, no solo teórico sino vivencial, de las elaboraciones tradicionales base. Se destaca, en ese sentido, la postura de algunos chefs jóvenes que han sabido proyectarse hacia la cocina tradicional, para aprehender sus características esenciales y reelaborarlas en nuevas preparaciones, siempre bajo un perfil de trabajo investigativo y de respeto hacia sus particularidades culturales.

Al respecto, el chef Diego Gutiérrez opina que, bien o mal, los jóvenes cocineros están haciendo algo distinto de lo que ya se ha hecho tradicionalmente; considera, asimismo, que de la tradición se produce la vanguardia y la renovación y que, de no presentarse este proceso de innovación, habría estancamiento (Padilla, 2019b., párr.9). En esto, Gutiérrez coincide de cierta manera con el chef guayaquileño Miguel Ponce, para quien "la gastronomía ecuatoriana está en un momento innovador, en el que los cocineros jóvenes trabajan con la mentalidad de innovar y crear conceptos" (Beltrán, 2015, párr. 6).

Esto no quiere decir que abandonen la tradición gastronómica ecuatoriana. Al contrario, muchas de sus propuestas se fundamentan en un proceso de investigación variado, que no se basa sólo en la revisión bibliográfica, sino que se alimenta también de fuentes provenientes de la tradición oral (entrevistas, etc.). Es importante tomar nota de ese esfuerzo, ya que da muestras de que es sólo a través de la generación de un punto de partida sólido que se puede diseñar una propuesta asertiva y representativa de una cultura gastronómica tradicional que, pese a los cambios y renovaciones, mantenga viva la cocina tradicional.

Así pues, la cocina tradicional tiene muchas cosas que enseñar. Pero no sólo eso. Es necesario comprender su revitalización como una responsabilidad moral y profesional de los gastrónomos ecuatorianos. Sólo comprendiendo que las libertades de la creatividad no son prácticas contrarias al respeto de los sabores tradicionales, se podrá generar nuevas propuestas asertivas, que representen al mismo tiempo la riqueza de la cocina local y nacional.

\section{BIBLIOGRAFÍA}

Beltrán, J. (10 de abril de 2015). Las nuevas tendencias de la cocina ecuatoriana se muestran en un festival de Cuenca. El Comercio. Recuperado de https://www.elcomercio.com/actualidad/cuenca-azuay-gastronomia-alimentos-chefs.html

Bustamante, O, \& Cabrera, D. (2014). Cambios en los patrones alimentarios de los habitantes del barrio el vergel Cuenca-Ecuador (tesis de grado). Recuperado de http://dspace.ucuenca.edu.ec/ handle/123456789/20554

Cordero, M. (2018). Cuenca, Memoria y Cocina. Cuenca: Editorial Don Bosco-Centro Gráfico Salesiano.

Padilla, M. (2019a). [Entrevista realizada a doña Nydia Vázquez de Fernández de Córdova, autora de Ashanga]. Entrevista inédita.

(2019b). [Entrevista realizada al chef Diego Gutiérrez, propietario del restaurante La caleta]. Entrevista inédita.

Sotomayor, D. (2012). Historia de la cocina cuencana en la segunda mitad del siglo XX (Tesis de grado). Recuperado de http://dspace.ucuenca.edu.ec/bitstream/123456789/1596/1/tgas59.pdf

Unigarro, C. (2010). Patrimonio cultural alimentario. [Versión PDF]. Recuperado de https://biblio.flacsoandes.edu.ec/catalog/resGet.php?resId=52870

Vera, D. (2016, 29 junio). En Cuenca aún se conserva la tradición de reunirse en familia para almorzar. El Telégrafo. Recuperado de https://www. eltelegrafo.com.ec/noticias/septimo/1/en-cuenca-aun-se-conserva-la-tradicion-de-reunirse-en-familia-para-almorzar

Vintimilla de Crespo. (1993). Viejos secretos de la Cocina Cuencana. Cuenca: Imprenta y Offset Atlántida. 


\title{
“ASOMBRADOS” ESCUCHA A LA INFANCIA A TRAVÉS DE LA OSCURIDAD
}

\author{
${ }^{1}$ Licenciada en Ciencias de la Educación Inicial \\ cintyadaniela.cantosalvarez@yahoo.com \\ (iD https:/orcid.org/0000-0002-5724-5688 \\ ${ }^{2}$ Licenciado en Ciencias de la Educación Inicial \\ jonnnna@outlook.com \\ (iD https://orcid.org/0000-0002-8438-9580
}

Cintya Daniela Cantos Alvarez ${ }^{1}$, Jonnathan Joel Paida Sánchez ${ }^{2}$

\author{
Fecha de recepción: \\ Fecha de aceptación:
}

\section{RESUMEN}

El trabajo titulado "Asombrados Escucha a la Infancia a través de la Oscuridad", busca trabajar la escucha a la infancia para la reinterpretación el Currículo Nacional de Educación Inicial 2014, partiendo de la documentación pedagógica de las acciones de juego, expresiones y producciones de los niños y las niñas en ambientes tenues estéticamente trabajados conjuntamente, desde casa, con los padres de familia del Centro de Educación e Innovación anexo a la UNAE en la ciudad de Azogues-Ecuador. Se emplea una metodología de corte cualitativo, con un posicionamiento semiopráctico en la investigación con los niños y no de los niños, observando y documentando pedagógicamente sus formas de hacer en la oscuridad para dejarnos sorprender y asombrar por la infancia. Reflexionando nuestras acciones con la infancia desde la manera en cómo se interpreta el Currículo de Educación Inicial 2014 que da cuenta de la imagen que el docente tiene de niño y niña. Finalmente, se puede expresar que para escuchar a la infancia debemos interactuar bajo relaciones interculturales y no intergeneracionales, bajo un acercamiento sensible de escucha en complicidad con la oscuridad, para propiciar experiencias significativas que les permitan conocer y ver el mundo con otros ojos.

Palabras Clave: escucha a la infancia, documentación pedagógica, Currículo de Educación Inicial 2014

\begin{abstract}
The work entitled "Astonished Listens to Children through the Darkness", seeks to work on listening to children for the reinterpretation of the National Curriculum for Initial Education 2014, based on the pedagogical documentation of children's play actions, expressions and productions and the girls in aesthetically dim environments worked continuously, from home, with the parents of the Education and Innovation Center annexed to the UNAE in the city of Azogues. A qualitative methodology is used, with a semi-practical positioning in research with children and not with children, observing and pedagogically documenting their ways of doing in the dark to allow us to be surprised and amazed by childhood. Reflecting on our actions with children from the way in which the 2014 Initial Education Curriculum is interpreted, which accounts for the image that the teacher has as a boy and a girl. Finally, it can be expressed that in listening to children we must interact under intercultural and not intergenerational relationships, under a sensitive approach of listening in complicity with the dark, to promote meaningful experiences that will allow them to know and see the world with different eyes.
\end{abstract}

Keywords: listening to childhood, pedagogical documentation, Curriculum of Initial Education 2014

Forma sugerida de citar: Cantos Alvarez, C. D. \& Paida Sánchez, J. J. (2020). Asombrados" Escucha a la infancia a 


\section{INTRODUCCIÓN}

$\mathbf{E}$ sta investigación está direccionada a resaltar la importancia de escuchar a la infancia atendiendo a sus intereses y "formas otras"1 de pensar, conocer y percibir el mundo, de una manera distinta a lo establecido. Escuchar demanda estar atentos a los sonidos que producen "otros". Esto implica percibir lo que nos quieren expresar con palabras, gestos, sentidos y movimientos. En primer lugar, se parte de un escenario de confinamiento por la emergencia sanitaria que se vive en el mundo y que ha puesto en evidencia las voces silenciadas de los niños y niñas por los adultos. Pareciera que, en esta situación, lo más importante es el cumplimiento del currículo, "avanzar" y no escuchar a los niños y niñas ni a sus intereses. En segundo lugar, esta investigación es el resultado de un proceso que inicia en séptimo ciclo con la implementación del ambiente de aprendizaje "Aula oscura Asombrados"; y que ahora se retoma de dicha vivencia el trabajo en un ambiente no habitual.

El ambiente de aprendizaje "Aula oscura Asombrados" se concibió en un ciclo anterior como un espacio en respuesta a los intereses y gustos de los niños y niñas, los cuales se han podido detectar durante el transcurso de las prácticas pre-profesionales de los últimos 4 ciclos de formación. Es un aula pintada de negro y oscurecida totalmente, dentro de la cual se organizan espacios de aprendizaje los cuales cuentan con materiales que son comunes en el hogar, por ejemplo: linternas, teselas, harina, cernidores, tubos de papel higiénico, entre otros y de acuerdo a ello se ha asignado un recurso principal, estos son; la luz: luz negra, luces led, caja de luz, luz natural, sonido: luces audiorítmicas, sensores de sonido y parlantes, los cuales están vinculados entre sí y posibilitan recorridos dentro del espacio.

Ahora bien, este proyecto ha buscado trabajar la escucha mediante experiencias en un espacio particular antes en el ambiente de aprendizaje "Aula oscura Asombrados" y ahora adecuado por los padres en sus casas tuvo una especificación concreta, el espacio debía ser tenue, el diseño del espacio dependió de la perspectiva de infancia que tienen los investigadores, de la experiencia de los proyectos realizados en las prácticas pre profesionales durante su formación y de las condiciones reales de los hogares, en este sentido, lo que se hizo fue posibilitar que los niños y las niñas jueguen en un ambiente de semioscuridad.

Entendiendo a la infancia como una cultura con sus propias maneras de hacer, conocer y saber, el espacio sugirió de la adecuación con objetos y materiales que se tenían en casa y que se prestaran para jugar con la luz y la sombra causando sensación de "asombro" en los niños y niñas y a su vez permitan trabajar el currículo de Educación Inicial de forma indirecta. Se escogieron del currículo las habilidades a trabajar, pero también lo que nosotros buscamos generar, es decir la intencionalidad de nuestra propuesta estuvo basada en la exploración. Porque la infancia "es una manera de estar y vivir que, para quererla, no necesita que se la comprenda" (Vilanova, 2014) solo necesita ser escuchada atendiendo a los intereses, preferencias, emociones y expresiones de los niños y las niñas, dando voz a esos cuerpos que tienen muchas historias para contar.

Trabajar en la oscuridad "propicia un hacer y mirar de distintos, extraños o contrarios a lo establecido" (Vilanova, 2014). Es extraordinario ver cómo ese cambio de matiz (trabajo en ambientes oscuros) saca a flote las "formas otras" de actuar y desenvolverse que tienen los niños, pero que la escolarización ${ }^{2}$ los limita encasillándoles en destrezas establecidas que deben cumplir de una misma manera sin tomar en cuenta la diversidad y los "otros saberes" o "formas de hacer" que tienen las niñas y los niños.

\footnotetext{
1 "formas otras" (Vilanova, 2014) de conocer y vivir que tienen los niños y niñas, es la manera como hacen presencia los niños y las niñas en la escritura, generado desde las investigaciones con niñas y niños que ha impulsado Vilanova irrumpiendo en el texto lingüista y el discurso académico que no cesa de hablar DE los otros en lugar de hablar CON los otros, por lo que de ahora en adelante en este proyecto se escribirá así (entre comillas y de forma invertida, aunque no se cite a la autora continuamente cada vez que se use en concepto). De esta manera, se busca atender a la perspectiva de los investigadores que buscan acercarse a la manera en la que los niños usan las palabras para pensar su vida y el mundo.

${ }^{2}$ Desde nuestra experiencia en la mayoría de CEI Y CIBV donde hemos realizado nuestras prácticas pre-profesionales, hemos evidenciado que se escolariza totalmente a los niños, siguen una misma estructura de trabajo que corresponde a la metodología tradicional sin importar la diversidad, aunque dentro de los currículos se supone que se trabaja desde el constructivismo, pero la realidad refleja otra cosa.
} 
Se asume un posicionamiento semiopráctico ${ }^{3}$, entendiendo como la acción viva de la investigación que rompe con las estructuras establecidas a nivel metodológico y de discurso, y donde conecta la investigación con la manera como se ha constituido, en sí mismo, el investigador. Por lo que se trabaja con herramientas reflexivas como la observación con propósito de escucha y atención, la documentación pedagógica para recordar, reconstruir y narrar la experiencia, también, como forma de comunicación tanto para los investigadores como para quienes han sido parte de la investigación (padres, docentes, y tutores), generando posibilidades de reflexión y aprendizaje. La narración de la experiencia se ha inclinado hacia el relato estético con el fin de que pueda dar cuenta de lo acontecido y que provoque sensaciones particulares en nosotros y en ustedes, los lectores.

Se escribe este texto desde una narrativa semiopráctica como constancia del posicionarnos en relación a la Infancia, como forma de narrar las relaciones vividas generadas durante esta investigación en sentido de ESTAR con los niños y hablar CON ellos y no DE ellos.

\section{METODOLOGÍA}

Se parte desde el sentido mismo de la experiencia como investigación educativa donde "la manera de entender la investigación es consustancial con la manera de entender la educación" (Contreras \& Pérez, 2010, pág. $70)$, en este caso específicamente hacerla con la mirada que tenemos de Infancia(S) en el sentido de investigar CON los niños y no SOBRE los niños, desde "el efecto estético de una ética del investigar que recorre caminos sin esconder sus pasos porque no necesita exponer evidencias ni demostrar datos" (Vilanova, 2019, pág. 15). Nos guiamos por el posicionamiento investigativo de la semiopraxis mediante el cual se rompe estructuras metodológicas y de discurso hegemónico centrado en discursos lógicos que ocultan las relaciones entre cuerpos, desde la semiopraxis que distingue "discurso sobre el cuerpo" y "discurso de los cuerpos" (Grosso, 2008, pág. 232) como acto vivo de relaciones con el "otro".

Esta investigación es de corte cualitativo, desde un paradigma crítico-interpretativo, puesto que parte de la concepción holística de los participantes como un todo, en este caso la infancia(S), la interacción se da de forma natural donde el investigador es parte de la investigación. Desde este enfoque el docente investigador cualitativo es un actor social que participa e interactúa en el contexto donde realiza la investigación, generando una relación con los niños y las niñas y con el contexto donde realiza la investigación (Cerrón, 2019).

Mediante procesos de reflexión de la realidad social en torno a la escucha a la Infancia(s) y la construcción de imagen que se ha realizado de niño, reflexionando o reinterpretando el currículo de Educación Inicial desde una perspectiva infancia que reconoce sus maneras de hacer y saber de manera respetuosa. A través de la reflexión se pretende llegar a una transformación del pensamiento de los investigadores a medida que va transcurriendo la investigación al sorprenderse con los hallazgos. Reflexionar desde el sentido de SER docente que va más allá de lo preestablecido, desde el sentido mismo de hacer investigación en Infancia (Melero, 2012).

Esta investigación realiza una narración de los hechos como parte de lograr transformar prácticas educativas, que se apeguen al respeto y reconocimiento de los niños y niñas como una cultura infantil. Para ello es necesario que se visualicen las formar de hacer que tienen los niños en este caso en un ambiente oscuro que logra conectarse con ellos y sus intereses, que en complicidad con la oscuridad se pueda fracturar lo preestablecido o sobreentendido de la infancia. Partiendo de esta idea se hablará con los niños, se presentarán fotografías de ellos con sus nombres y narraciones permitiendo interpretar las situaciones y los momentos vividos lo que nos lleve a pensar o repensar críticamente la educación inicial en un marco de respeto a la Infancia(S).

Se trabajó mediante la Investigación Acción Cooperativa (IAC), en el sentido de implementar la propuesta del trabajo en la oscuridad desde la cual se va generando la documentación, narración, análisis de la experiencia y reinterpretación del currículo nacional de educación Inicial 201. La investigación se realizó coope-

${ }^{3}$ Esta investigación pretende trabajar desde la semiopráctica para contextualizar con la experiencia educativa que implica trabajar con la infancia, y la fotografía como un proceso propio de la investigación en este campo. Haciendo referencia a (Vilanova, 2014) y (Grosso, 2017) 
rativamente entre la pareja practicante, padres de familia, niños y docentes del centro. Como menciona Rincón, 1997 citado en Bausela (2003) "Reflexionar antes de la acción (planificar), durante la acción (opciones en la acción) y después de la acción (sobre los efectos de las acciones y posterior revisión de la acción planificada). Planificar de forma intencional acciones de mejora" (pág. 122). Se basa en la autorreflexión y construcción de una mejora del mismo investigador como de la practica educativa, desde el acercamiento y construcción de una relación con los niños y las niñas mediante las interacciones cercanas que permitan dar voz a esos cuerpo negados o silenciados por el egocentrismo adulto, desde un enfoque semiopráctico como estrategia o táctica para la deconstrucción de la hegemonía como lo dice Grosso (2017) para "devolver al pensamiento, y enfrenta a las ciencias sociales, a un reconocimiento dialógico más amplio de los lugares de enunciación y las interacciones discursivas" (pág. 87).

El proceso metodológico que se describe en el desarrollo se lo realiza por fases puesto que, está pensado como propuesta pedagógica ya que sigue los pasos que se han realizado para consolidar esta investigación.

\section{DESARROLLO}

\section{FASES DE LA IAC:ELEMENTOSY REFLEXIONES PARA LA PROPUESTA PEDAGÓGICA}

\section{Primera fase: Práctica}

Se considera el inicio de la propuesta como es el diseño de las sesiones de trabajo, los registros, sistematización y seguimiento de los encuentros. Se establecen horarios de conexión mediante plataformas tecnológicas como ZOOM en horas ajustadas a necesidades de los representantes de los niños, se acordó 40 minutos pasando un día a las $6 \mathrm{pm}$. Los participantes fueron 27 niños y niñas de 3 a 6 años.

El registro de las sesiones se llevó mediante los diarios de campo, videos de los encuentros por ZOOM, reflexiones entre la pareja practicante y la matriz de sistematización de experiencias. Las anotaciones de los diarios de campo guiaron la construcción de la sistematización, los videos permitían recoger observaciones sobre los intereses de los niños para plantear las siguientes actividades. Las actividades iban en de acuerdo a lo que iba surgien- do en la anterior. La documentación se dio por parte de los practicantes, pero también por parte de los padres o acompañantes de los niños.

Se orientó mediante dos dibujos como ejemplos de construir sus espacios oscuros pero los padres utilizaron su creatividad y mobiliario disponible para construirlos. Al inicio cada niño y niña tenía su "cuarto oscuro" especifico donde realizábamos las actividades, pero se empezó a necesitar más espacio o eso pudimos percibir por parte de los niños puesto que ya no usaban los espacios construidos únicamente, sino que acondicionaron todo un cuarto para trabajar, al momento de los encuentros cerraban las cortinas, apagaban las luces, cerraban la puerta, se ubicaban en un espacio libre de objetos y que estuviera una pared cerca, los niños y las niñas habían solitos diseñado o pensado su espacio de forma funcional para ellos.

Las actividades al inicio estaban encaminadas hacia una destreza del currículo, pero al realizar se salía, iba más allá, pasaba el límite que establecía la destreza así que se pasó a segundo plano el currículo porque creíamos y veíamos la capacidad de los niños para hacer, las actividades se prestaban para que cada niño y niña haga a su manera y de manera autónoma, desde el inicio de las actividades demostraron curiosidad e inclinación por los cuentos, sombras con: manos, cuerpo, siluetas y objetos de su hogar, exploración con materiales.

Los cuentos o más bien narraciones surgían de la creatividad y vivencia que cada niño y niña tenía cada uno inventaba las historias en ese momento y compartía con todos sus compañeros, otras veces nosotros narrábamos el cuento y ellos dibujaban lo que les gustaba o realizaban sombras de las acciones o personajes utilizando su cuerpo y realizando sonidos que acompañaban las representaciones.

Las actividades iban surgiendo de los intereses de los niños y las niñas al escucharles con todos los sentidos podíamos percibir señales de disfrute en ellos al realizar alguna acción y eso nos servía para preparar la siguiente actividad de manera que responda a sus gustos. Escuchar ha permitido acercarnos a la infancia para respetar su cultura para construir y fortalecer la imagen de niño que tenemos y para adentrarnos en el currículo de Educación Inicial para reinterpretarlo desde otra perspectiva, desde una mirada de trabajo en un ambiente oscuro e infancia(s). 
Segunda fase: Análisis de la práctica a partir de lo que se escucha, se silencia y se potencia para la construcción de imagen de niño

En cuanto al diseño de sesiones, sistematización y seguimiento, resultados de la propuesta y revisión documental del currículo de Educación Inicial 2014. Durante los encuentros manteníamos diálogos con los niños como si estuviésemos hablando con otro adulto y todo fluía muy bien, no encontramos por qué en los centros se infantiliza a los niños, porque solo tratarle de "mi amorcito", "mi chiquito" o no utilizar algunas palabras porque "ellos son chiquitos y no entienden" o decir que a ellos les gusta "cosas alegres porque son niños" (hablarles con exaltación), desde nuestra perspectiva no todos los niños y niñas son iguales, no porque eres niño siempre te gusta que te hablen en tono de exaltación, no solo tienen el sentimiento de felicidad, si analizamos esas palabras o actitudes podemos darnos cuenta que refleja una imagen de niño inferior, con desconocimiento, incompleto.

Usualmente el aprendizaje se centra en lo que dice el currículo y las destrezas, se planifica para el cumplimiento de esa destreza y se limita a la expresividad puesto que todos tienen que hacer lo mismo no solo el resultado sino el proceso, actividades cerradas que no permiten las formas de hacer que tienen los niños y niñas la justificación es que deben hacerlo así, lo que demuestra una escucha al currículo y no a los niños y niñas, hay actividades que a los niños y niñas no les gusta y no desean hacerlo con convencimiento de la docente terminan haciendo no por gusto sino por obligación porque aunque pareciera que se escucha en realidad solamente se oye.

Los cuentos reflejan las experiencias de los niños y niñas, sus gustos, sus conocimientos su creatividad nos refleja una imagen de niño co-constructor, capaz de expresarse e inventar una historia y no solo capaz de repetir algo. Sus relatos inventados tienen un principio, un desarrollo y un final que nuevamente nos presentan a un niño muy capaz.

Las acciones de juego con las sombras reflejan un niño con dominio corporal, con coordinación entre la linterna y sus movimientos, un niño imaginativo capaz de realizar con sus manos la sombra de un animal y no es que lo han conseguido porque les hemos dicho como lo hagan, lo han hecho porque son capaces, porque no están vacíos, si puede sonar extraño estos comentarios que hemos podido ver y escuchar en esta propuesta, pero también hay que pensar cuantas veces dejamos SER y HACER a los niños. Talvez no hemos visto porque no hemos generado espacios de escucha, observación y documentación.

Durante el desarrollo de la propuesta se ha insistido a los padres en que tomen una foto de los niños cuando estén jugando el ambiente tenue de algo que les llame la atención y lo han tomado como evidencia del trabajo o evaluación, para nosotros una fotografía es dejar huella de las acciones que hacen los niños, es permitirnos asombrarnos con las acciones de los niños.

El currículo proyecta la imagen de un niño inmaduro que necesita cumplir ciertas destrezas para prepararse para el siguiente año escolar, un niño con un límite que va de acuerdo a la edad, el currículo si bien es de ayuda para los docentes también genera violencia simbólica.

En esta pandemia con la propuesta hemos escuchado los intereses de los niños y niñas, sus sentimientos y gustos, modificado en algo la imagen que los padres tenían de sus hijos, y el sentido de SER docente y rol de investigador porque hemos vivido la investigación y contextualizado a la infancia.

En este punto analizaremos como se interpreta el currículo en los centros educativos donde hemos realizado prácticas pre profesionales a lo largo de nuestra formación. En general el currículo es una camisa de fuerza que tiene la misma perspectiva de interpretación en la mayoría de centros a excepción del CEI UNAE que tiene un modelo pedagógico innovador.

De manera general en los centros que atienden a niños de 1 a 5 años en los que se han realizado las practicas pre-profesionales hay una repetición de acciones las cuales silencian a los niños, esto se debe a una interpretación común del currículo, se planifica por cumplir requisitos, las planificaciones no se ejecutaban en los centros con niños de 1 a 3 años se manejan como guarderías, la jornada diaria era: ingreso, desayuno, ir a la sala ver una película, refrigerio, patio, aseo personal pero lo realizaba la educadora a cada niño y el resto esperaba, almuerzo(algunos niños ya se iban), ir a la sala a dormir, refrigerio y salida. En cuanto a otro punto que establece el currículo los ambientes o rincones de aprendizaje se 
organizan sin intencionalidad, literalmente los rincones están ubicados en un rincón pegado el nombre y sin objetos porque los niños desordenan.

En los centros que atienden a niños de 3 a 5 años las actividades estaban casi en su totalidad enfocadas en el trabajo en láminas, actividades muy estáticas con una misma estructura, los rincones de aprendizaje se mantenían como en el caso anterior sin material o escaso, no se usaba como parte del proceso de enseñanza sino como entretenimiento de los niños cuando las maestras tenían reunión.

\section{Tercera fase: Interpretativa}

En esta fase narraremos la vivencia de ESTAR con los niños y niñas y ser espectadores de sus formas de hacer en sus "cuartos oscuros" asi lo llamaron los niños y niñas al espacio que se construyo.

\section{Ilustración 1 » Sombras con manos. Archivo de la investigación.}

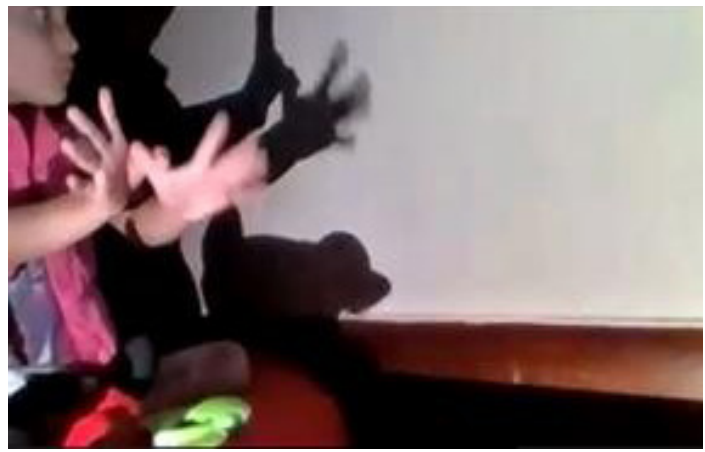

Valentina realiza la sombra de una paloma y su mirada se centra en la sombra que proyecta en la pared. Hace sus movimientos para que la paloma vuele, pero siempre concentrada a la sombra.

Ilustración 2 » Organización de escenas. Archivo de la investigación.

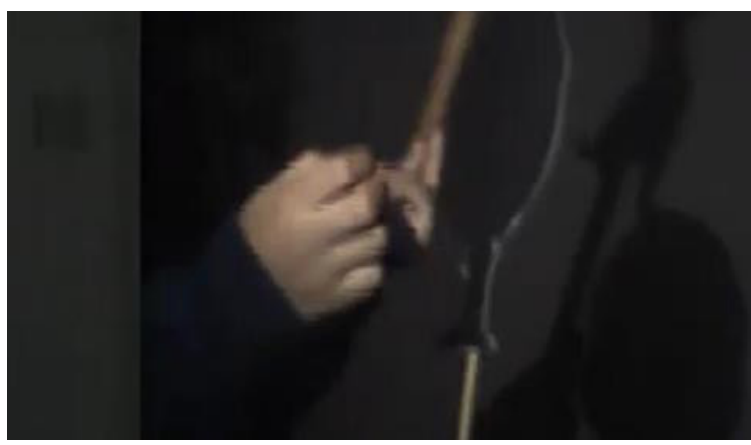

Es Rafael y su cuento trata de un "lobo que tenía hambre y se comió un gato, le persiguió a un caracol para comérselo también" su narración la acompañada por sonidos de los animales, en la foto se visualiza la sombra de un "lobo que se subió en un árbol a mirar la luna y aullar". Es impresionante su capacidad de creatividad y como coordina la ubicación de sus manos para proyectar la sombra de la escena.

Ilustración 3 » Pensamiento complejo. Archivo obtenido de la madre del niño.

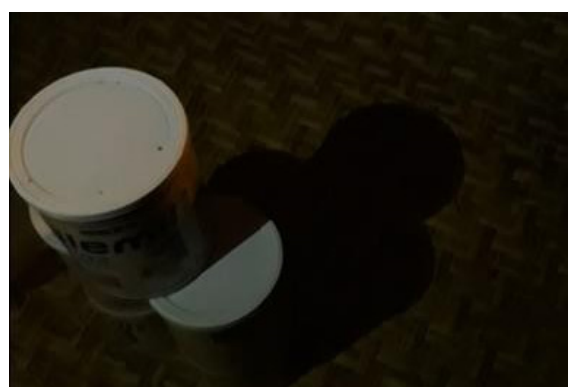

Es la construcción y sombra de Roberto nos comenta que hizo la sombra de una botella de perfume con los tarros de leche de su hermanito.

\section{Ilustración 4» Mi cuento dibujado. Archivo obtenido de} la madre de la niña.

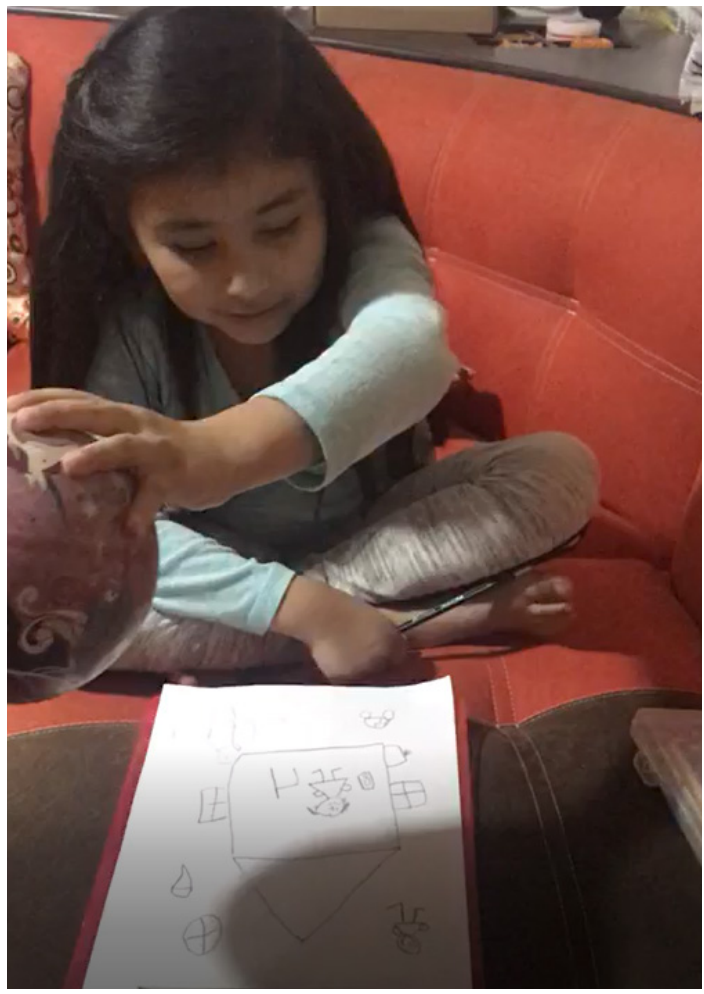


Es Brithany y nos relata un cuento con la sombra de su objeto preferido que es una pelota, ella ha dibujado su cuento, mientras va narrando no quita su mirada del dibujo e incorpora a su dibujo la sombra de una pelota que forma parte de la narración.

\section{Ilustración 5» Explorando con la pantalla. Archivo de la investigación.}

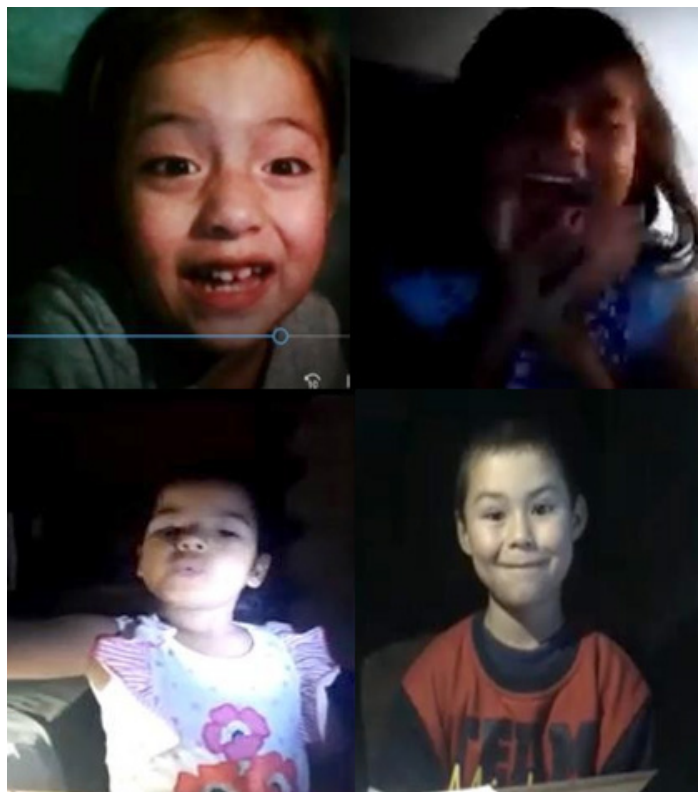

Martina, Anahí, Karly y Milán haciendo muecas mientras se miran en su pantalla, creemos que lo hacían cuando se conectaba desde el celular y solo podían verse a sí mismos por lo que jugaban con su rostro, son fotos de días distintos.

\section{Cuarta fase: Semiopraxis e Infancia(s)}

"Asombrados" buscó generar una escucha a esas "formas otras" de hacer y crear de la infancia, en pequeños espacios donde niños y niñas se conecten con sus maneras de hacer y pensar, poco escuchadas y por lo tanto poco valoradas por la cultura adulta y que, en este caso, se despierta directamente bajo asombro en sus padres a través de un trabajo al estilo "homeschooling" (Illich, 1970; Stern, 2009) ${ }^{4}$ ESTAR con los niños y niñas nos lleva complejizar nuestro pensamiento, sus acciones nos sorprenden porque rebasan los límites de lo preestablecido de un niño que tiene que superar etapa de la infancia y llegar al ideal ser adulto.

Trabajar en un ambiente oscuro que rompe con los esquemas o ciertos prejuicios del temor a la oscuridad y de lo colorido que es todo en los centros educativos ya hacía la diferencia, teníamos otro modo de acercamiento a la infancia por ende generaba otras relaciones, respetuosas y sin ejercer poder. Queríamos trabajar las destrezas del currículo y lo hicimos de manera indirecta porque al inicio las escogimos, recordamos que las del ámbito de expresión y comprensión del lenguaje estaban muy limitantes, el caso de "Expresarse utilizando oraciones cortas en las que puede omitir o usar incorrectamente algunas palabras" (MINEDUC, 2014, pág. 37). Se mantenían diálogos fluidos y largos, otra destreza "Relatar cuentos, narrados por el adulto, manteniendo la secuencia, sin la ayuda del paratexto" (MINEDUC, 2014, pág. 37). Por qué hacer que repitan cuando ellos pueden crear sus propios relatos por qué opacar su imaginación y creatividad sino al contrario potenciarla.

Inicialmente se pensó hacer actividades variadas en el cuarto oscuro, pero en el camino de escuchar a la infancia se tomo el rumbo de hacer lo que los niños y las niñas querían hacer porque como docentes nosotros estamos para eso, para responder a sus intereses para propiciar la construcción de sus aprendizajes no para que hagan lo que nosotros queremos que hagan. Se direccionaron por los relatos y casi todas las actividades en torno a cuentos inventados por los niños, niñas y nosotros.

\section{Quinta fase: Discusión}

La imagen de niño que se proyecta en las escuelas donde hemos estado es un niño aburrido, etiquetado porque sale del canon establecido, son inquietos los llaman hiperactivos, pero como no estar inquieto si son niños y quieren jugar, moverse y explorar como no se les escucha su medio de expresión es poniendo "desorden" como señal de aburrimiento de que no les gusta lo que están haciendo, qué imagen de niño proyecto al tenerlos sentados por horas y horas.

${ }^{4}$ Hemos señalado a (Illich, 1970 )como iniciador del movimiento estudiar en casa (homeschooling) desde la desescolarización y a (Stern, 2009) como como representante actual del homeschooling. 
Ilustración 6» Modo de trabajo de centros. Archivo de los autores de la investigación.

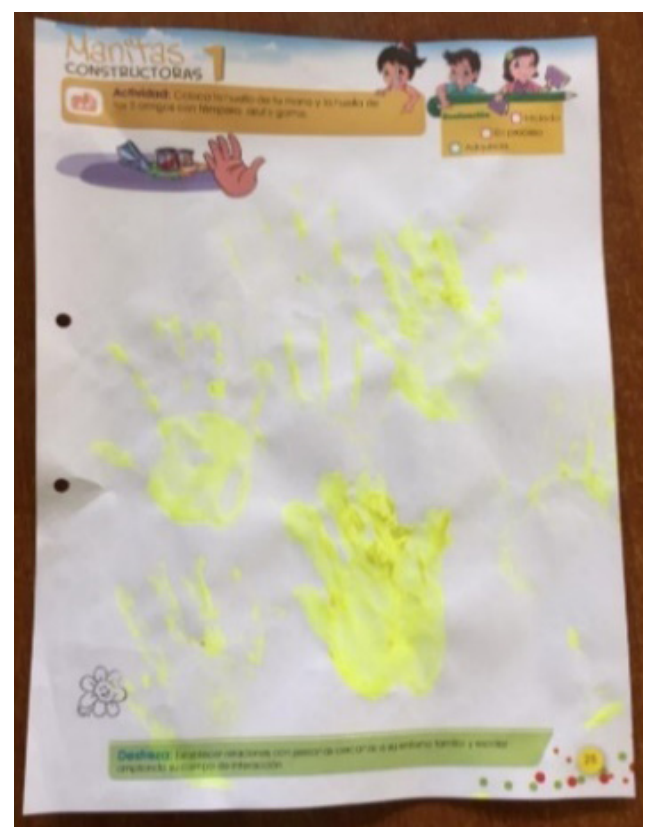

En esta foto se refleja la imagen de niño que se proyecta en la escuela porque muchas de estas laminas son la huella de lo que han hecho durante su estancia en los centros educativos, laminas que generan frustración en los niños y niñas porque no quieren hacerlo, porque no lo disfrutan, porque sub estiman sus capacidades.

Los centros educativos en su mayoría son "fabricas" donde moldean el niño futuro adulto que la sociedad requiere, ven a la infancia como una etapa que debe ser superada, incluso se escucha frases como "pareces niño" o eso es "infantil" es un cumplido o es una forma de inferiorizar a la infancia. La imagen de niño que hemos visto en la mayoría de centros de práctica es un niño incompleto e incapaz que necesita que le controlen.

Ilustración 7»Expresiones repetitivas de los niños en centros. Archivo de los autores de la investigación

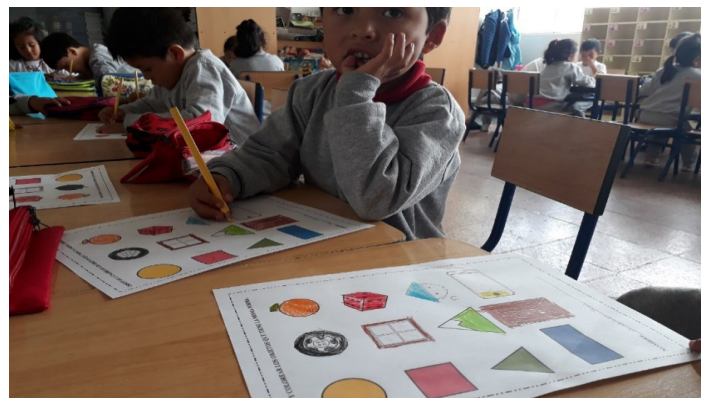

No se les escucha, no importa sus intereses lo que importa es llenar una carpeta con muchas láminas de trabajo porque no importa el proceso sino el resultado, importa la motricidad fina que se refleje en pintar sin salirse de los bordes, así es la única manera de hacerlo o hay otras maneras. Para el ámbito de expresión y compresión del lenguaje la única forma es utilizar los mismos cuentos y hacer que los niñas y niñas repitan acaso dudamos de su capacidad para crear en lugar de reproducir.

La mayoría de centros educativos cuentan con los mismos ambientes de aprendizaje y la misma estructura de materiales, rincón del hogar (cocina, mesas, sillas, utilería, cama, plancha, etc.); expresión artística (escarcha, hojas de papel bond, foami, pinturas, marcadores, acuarelas); lógico matemático (figuras geométricas, números, rosetas, bolas de colores, etc.); construcción (legos, plastilina); psicomotricidad (colchonetas, ulas, conos, pelotas, cuerdas), son de plástico (juguetes) quién determino que solo ese material se debe tener en las escuelas porque no tener algunas cosas como plancha de verdad aunque este dañada, por qué no variar el material tener maderas en diferentes formas y tamaños para la construcción, por qué no hacer que se extraigan pigmentos naturales, por qué no crear otros ambientes de aprendizaje, entonces bajo qué imagen de infancia están actuando los docentes.

Si interpretamos desde otra perspectiva el currículo es posible hacer un cambio sobre todo para respetar a los niños y que no se de relaciones de poder en los centros educativos entre docentes y niños y niñas sino una relación cultural, mas no intergeneracional como dice Kohan (2007) tratemos a los niños y niñas como si fuesen extranjeros, con respeto y curiosidad por acercarnos a eso que no conocemos, a un extranjero no le obligamos a habar nuestro idioma o hacer lo que nosotros hacemos o si, a un extranjero lo acercamos a nuestra cultura de manera respetuosa sin imposiciones, sería lo mismo con los niños un intercambio los extranjeros de nuestra vida.

Esta interpretación del currículo se ha mantenido desde hace algunos años son prácticas que se replican, ahora bien de dónde surge esa interpretación, tal vez el sistema debe cambiar, la sociedad en si a construido esa imagen de niño pero quién es el responsable de proyectarla, será la escuela con sus acciones, entregar al final de año escolar una carpeta llena de láminas refleja esa imagen de infancia negada y ocultada por esas hojas 
de trabajo, por qué ocultarla, por qué tal vez y rompa lo establecido, por qué desequilibre lo que tenemos. En nuestra opinión deberíamos vestigiarla y exponerla para asombrarnos con lo que podríamos encontrarnos, por que como dice Malaguzzi se debe dejar huellas de lo qué hacen y cómo lo hacen.

\section{CONCLUSIONES}

La escucha a la infancia debe darse en los centros educativos para acercarnos a los intereses de los niños y niñas en sí a su cultura para dejar de lado las relaciones de poder que silencian y generan violencia simbólica en las instituciones, si tenemos un acercamiento de relación cultural tendremos un acercamiento respetuoso con los niños y niñas.

En este caso utilizamos como medio de acercamiento a los niños el trabajo en un ambiente poco habitual como es la oscuridad porque el desenvolvimiento del niño se ve influenciado de los estímulos que se le presenten en su ambiente, la oscuridad es una forma de atraer a los niños a explorar el mundo con otros ojos, bajo otras circunstancias que permitan a los niños sentir curiosidad.

La experiencia de documentar nos ha permitido concluir que la documentación pedagógica se debe incorporar a la práctica de los docentes esta permite procesos de reflexión sobre las acciones con los niños para mejorar el accionar en beneficio de la infancia, pero también permite reconocer y visibilizar las acciones de los niños y niñas en el proceso de construcción de su aprendizaje, tanto para el docente, niños, niñas y comunidad la documentación deja huellas que proyectan una imagen de infancia reconocida y no ocultada.

El currículo es un documento flexible que se lleva a la práctica mediante la interpretación del docente, por ello es importante que cada docente tenga conciencia del sentido de estar con los niños y de la imagen que tiene de niño porque esto influye en sus acciones y de ello depende como organiza los espacios y actividades.

\section{BIBLIOGRAFÍA}

Basuela, E. (2003). La investigación cooperativa, una modalidad de la invetigación-acción. Revista de Psicodidáctica(15-16), 121-130. Obtenido de https:// www.redalyc.org/pdf/175/17515081009.pdf
Cerrón, W. (2019). La investigación cualitativa en educación. Horizonte de la Ciencia, 9(17). doi:https://doi. org/10.26490/uncp.sl.2020.4.1.510

Contreras, J., \& Pérez, N. (2010). Investigar la experiencia educativa. Investigar la experiencia educativa. Madrid, España: Morata.

Grosso, J. L. (2008). Semiopraxis en contextos interculturales poscoloniales. Cuerpos, fuerzas y sentidos en pugna. Espacio Abierto, 17 (2), 231-245.

Grosso, J. L. (2017). En otras Lenguas: Semiopraxis popular - intercultural - poscolonial como praxis crítica. Azogues, Ecuador : UNAE.

Grosso, J. L. (2017). Más allá (más acá) de la fenomenología. En J. L. Grosso, En otras lenguas: semiopraxis popular-intercultural-poscolonial como praxis crítica (págs. 87-134). Azogues: Universidad Nacional de Educación. Obtenido de http://repositorio.unae. edu.ec/handle/56000/543

Illich, I. (1970 ). La sociedad descolarizada (Vol. Primera edición). Argentina: Godot.

Kohan, W. (2007). Política, educación y filosofía: La fuerza de la extranjeriedad. En W. Kohan, Infancia, política y pensamiento: ensayos de filosofía y educación. (págs. 9-17). Buenos Aires: Del estante.

Melero, N. (2012). El paradigma crítico y los aportes de la investigación acción participativa en la transformación de la realidad: un análisis desde las ciencias sociales. Cuestiones pedagógicas(21), 339-355. Obtenido de https://idus.us.es/bitstream/handle/11441/12861/file_1.pdf?sequence=1\&isAllowe$\mathrm{d}=\mathrm{y}$

MINEDUC. (2014). Curriculo de Eduación Inicial. Obtenido de https://educacion.gob.ec/wp-content/uploads/downloads/2014/06/curriculo-educacion-inicial-lowres.pdf

Stern, A. (2009). Yo nunca fuia la escuela. España: literalibros.

Vilanova, M. (2014). Descender desde la infancia: El desarrollo y el discurso de los "niños" ante "formas otras" de conocer yvivir. Infrome de investigación del Doctorado, Universidad de Barcelona, Barcelona. Obtenido de http://diposit.ub.edu/dspace/handle/2445/64964?mode=full

Vilanova, M. (2019). Infancia(S) del investigar o en busca del espacio. Mamakuna(10), 8-17. 